FORMAL AND ANALYTIC SOLUTIONS

OF DIFFERENTIAL AND DIFFERENCE EQUATIONS

BANACH CENTER PUBLICATIONS, VOLUME 97

INSTITUTE OF MATHEMATICS

POLISH ACADEMY OF SCIENCES

WARSZAWA 2012

\title{
CONVERGENCE OF FORMAL SOLUTIONS \\ OF FIRST ORDER SINGULAR PARTIAL DIFFERENTIAL EQUATIONS OF NILPOTENT TYPE
}

\author{
MASATAKE MIYAKE \\ Graduate School of Mathematics, Nagoya University \\ Furo-cho, Chikusa, 464-8602 Nagoya, Japan \\ E-mail:mmiyake@math.nagoya-u.ac.jp \\ AKIRA SHIRAI \\ School of Education, Sugiyama Jogakuen University \\ Hoshigaoka Motomachi 17-3, Chikusa, 464-8662 Nagoya, Japan \\ E-mail: shirai@sugiyama-u.ac.jp
}

Abstract. Let $(x, y, z) \in \mathbb{C}^{3}$. In this paper we shall study the solvability of singular first order partial differential equations of nilpotent type by the following typical example:

$$
P u(x, y, z):=\left(y \partial_{x}-z \partial_{y}\right) u(x, y, z)=f(x, y, z) \in \mathcal{O}_{x, y, z},
$$

where

$$
P=y \partial_{x}-z \partial_{y}: \mathcal{O}_{x, y, z} \rightarrow \mathcal{O}_{x, y, z} .
$$

For this equation, our aim is to characterize the solvability on $\mathcal{O}_{x, y, z}$ by using the $\operatorname{Im} P$, Coker $P$ and $\operatorname{Ker} P$, and we give the exact forms of these sets.

1. Introduction and result. Let $X=\left(x_{1}, x_{2}, \ldots, x_{n}\right) \in \mathbb{C}^{n}$. We consider the following first order nonlinear partial differential equation:

$$
\left\{\begin{array}{l}
\left(\sum_{i, j=1}^{n} a_{i, j} x_{i} \partial_{x_{j}}+c\right) u(X)=\sum_{i=1}^{n} b_{j} x_{j}+f_{2}\left(X, u, \partial_{X} u\right), \\
u(0)=0,
\end{array}\right.
$$

where $a_{i, j}, b_{j}$ and $c$ denote constants and $\partial_{x_{j}}=\partial / \partial x_{j}, \partial_{X} u=\left(\partial_{x_{1}} u, \ldots, \partial_{x_{n}} u\right)$.

We assume that the function $f_{2}(X, u, \xi)\left(\xi=\left(\xi_{1}, \ldots, \xi_{n}\right) \in \mathbb{C}^{n}\right)$ is holomorphic in a neighborhood of the origin in $(X, u) \in \mathbb{C}^{n+1}$ variables and an entire function in $\xi \in \mathbb{C}^{n}$

2010 Mathematics Subject Classification: Primary 35A20; Secondary 34M03, 34M25.

Key words and phrases: Convergence of formal solutions, Singular PDE, Nilpotent vector field. The paper is in final form and no version of it will be published elsewhere. 
variables with the following Taylor expansion.

$$
f_{2}(X, u, \xi)=\sum_{|\alpha|+p \geq 2} f_{\alpha, p}(\xi) X^{\alpha} u^{p},
$$

where $\alpha=\left(\alpha_{1}, \ldots, \alpha_{n}\right) \in \mathbb{N}^{n}, p \in \mathbb{N}$, and $X^{\alpha}=x_{1}{ }^{\alpha_{1}} \cdots x_{n}{ }^{\alpha_{n}}$ and $f_{\alpha, p}(\xi)$ denotes an entire function in $\mathbb{C}^{n}$.

Before stating the results, we prepare some notation and definitions.

Let $\mathcal{O}_{x_{1}}$ be the set of holomorphic functions at $x_{1}=0, \mathbb{C}\left[\left[x_{1}, x_{2}, \ldots, x_{n}\right]\right]$ be the set of formal power series of variables $\left(x_{1}, x_{2}, \ldots, x_{n}\right)$ over $\mathbb{C}, \mathcal{O}_{x_{1}}\left[\left[x_{2}, \ldots, x_{n}\right]\right]$ - the set of formal power series of variables $\left(x_{2}, \ldots, x_{n}\right)$ over $\mathcal{O}_{x_{1}}$ and $\mathcal{O}_{X}\left(=\mathcal{O}_{x_{1}, \ldots, x_{n}}\right)$ - the set of holomorphic functions at $X=0$.

Definition 1.1. For a formal power series $u(X)=\sum u_{\alpha} X^{\alpha} \in \mathbb{C}[[X]]$, we say that $u(X)$ belongs to the Gevrey class of order $s$, if the power series $\sum u_{\alpha} X^{\alpha} /|\alpha| !^{s-1}$ $\left(|\alpha|=\alpha_{1}+\ldots+\alpha_{n}\right)$ converges in a neighborhood of $X=0$.

For the equation (1), we know the following results (cf. GT], [MS1, [MS2, [H], O], $[\mathrm{S}$, etc.)

Theorem 1.2. Let $\Lambda=\left\{\lambda_{j}\right\}_{j=1,2, \ldots, n}$ be the set of eigenvalues of the matrix $A=$ $\left(a_{i, j}\right)_{i, j=1, \ldots, n}$.

(i) If $\Lambda$ satisfies the Poincaré condition, then the formal solution converges in a neighborhood of the origin (if it exists).

(ii) Let $\lambda_{j}=0$ for all $j=1,2, \ldots, n$. If $c \neq 0$, then the formal solution $u(X)$ exists uniquely, and it belongs to the Gevrey class of order at most $2 n$.

Theorem 1.2(i) is a very famous result for the theory of first order partial differential equations (cf. GT], MS1, MS2], [], etc.). On the other hand, when $\lambda_{j}=0$ for all $j=1,2, \ldots, n$, we say that equation (1) is of nilpotent type. In this paper, we shall study the solvability on $\mathcal{O}_{X}$ in the nilpotent case. In the case $c \neq 0$, by Theorem 1.2 (ii), the formal solution of (1) is not convergent in a neighborhood of the origin in general (cf. $[\mathrm{H}], \mathrm{O},[\mathrm{S}]$ ). Therefore, the purpose of this paper is to study whether the formal solution is convergent or not in the case when $c=0$.

In this paper, we study an example of equation of nilpotent type in $X=(x, y, z) \in \mathbb{C}^{3}$ of the form

$$
P\left(X, \partial_{X}\right) u(X):=\left(y \partial_{x}-z \partial_{y}\right) u(x, y, z)=f(x, y, z) \in \mathcal{O}_{X},
$$

or equivalently we study the kernel and cokernel of the mapping

$$
P\left(X, \partial_{X}\right): \mathcal{O}_{X} \longrightarrow \mathcal{O}_{X}
$$

In order to study this equation or the mapping, we express the function $g(x, y, z) \in \mathcal{O}_{X}$ or $\mathcal{O}_{x}[[y, z]]$ by

$$
g(x, y, z)=\left\{\sum_{i+2 j=\text { even }}+\sum_{i+2 j=\text { odd }}\right\} g_{i, j}(x) y^{i} z^{j}=: g_{\mathrm{e}}(x, y, z)+g_{\mathrm{o}}(x, y, z),
$$

where $g_{\mathrm{e}}$ and $g_{\mathrm{o}}$ denote the even part and the odd part of $g$, respectively.

Now our main result is stated as follows. 


\section{MAIN TheOREM 1.3.}

(i) Let $\operatorname{Im}\left(P ; \mathcal{O}_{X}\right)$ be the image of the mapping (4). Then $f(X) \in \operatorname{Im}\left(P ; \mathcal{O}_{X}\right)$ if and only if it satisfies $f(x, 0,0) \equiv 0$ and the infinitely many compatibility conditions:

$$
f_{2 n+2,0}(x)+\sum_{k=0}^{n} \frac{(2 k-1) ! !}{(2 n+1) ! !} D_{x}^{n+1-k} f_{2 k, n+1-k}(x) \equiv 0 \quad(n=0,1,2, \ldots),
$$

where $(-1) ! !=1$ and $(2 n+1) ! !:=1 \cdot 3 \cdots(2 n+1)$ and $D_{x}=d / d x$ denotes the differentiation in $x$. In other words, we have the following isomorphism for the cokernel $\operatorname{Coker}\left(P ; \mathcal{O}_{X}\right)$ of 4 :

$$
\operatorname{Coker}\left(P ; \mathcal{O}_{X}\right) \simeq \mathcal{F}:=\left\{f(x, y)=\sum_{n=0}^{\infty} f_{2 n, 0}(x) y^{2 n} \in \mathcal{O}_{x, y}\right\} .
$$

(ii) Let $\operatorname{Ker}\left(P ; \mathcal{O}_{X}\right)$ be the kernel of the mapping (4). Then we have

$$
\operatorname{Ker}\left(P ; \mathcal{O}_{X}\right) \simeq \mathcal{K}:=\left\{v(y, z)=\sum_{n=0}^{\infty} \sum_{k=0}^{n} C_{2 n-2 k, k} y^{2 n-2 k} z^{k} \in \mathcal{O}_{y, z}\right\},
$$

and the isomorphism is given by

$$
\mathcal{K} \ni v(y, z) \mapsto u(X)=\sum_{n=0}^{\infty} \sum_{k=0}^{n} C_{2 n-2 k, k}\left(y^{2}+2 x z\right)^{n-k} z^{k} .
$$

Theorem 1.3 will be proved by showing the unique solvability of the following Cauchy problem:

$$
\left\{\begin{array}{l}
P\left(X, \partial_{X}\right) u(X) \equiv f(X)(\bmod \mathcal{F}) \\
u_{\mathrm{e}}(0, y, z)=v(y, z) \in \mathcal{K}
\end{array}\right.
$$

2. Proof of Main Theorem 1.3. The proof of Main Theorem 1.3 will be done by the following plan.

1. We give the compatibility condition (6) or (7) in formal sense.

2. We give the condition (8) and property (9) in formal sense.

3. We prove the convergence of formal solution.

2.1. Research on $\operatorname{Im}\left(\boldsymbol{P} ; \mathcal{O}_{X}\right)$. We define the set of quasi-homogeneous polynomials $\mathcal{O}_{x}[y, z]_{p}(p \in \mathbb{N})$ by

$$
\mathcal{O}_{x}[y, z]_{p}=\left\{f_{p}(x, y, z)=\sum_{i+2 j=p} f_{i, j}(x) y^{i} z^{j}: f_{i, j}(x) \in \mathcal{O}_{x}\right\} \subset \mathcal{O}_{x}[[y, z]] .
$$

Then we easily see that

$$
P: \mathcal{O}_{x}[y, z]_{p} \rightarrow \mathcal{O}_{x}[y, z]_{p+1} \quad(p=0,1,2, \ldots) .
$$

Here after, $f_{p}(x, y, z)$ denotes the quasi-homogeneous polynomial of degree $p$. For $u(X)=\sum_{p \geq 0} u_{p}(x, y, z)$, we have $P u(X)=\sum_{p \geq 1} f_{p}(x, y, z)$. Therefore, in order that $f(x, y, z) \in \operatorname{Im}\left(P ; \mathcal{O}_{X}\right)$ it is necessary that $f_{0}(x, y, z) \equiv 0$, that is, $f(x, 0,0) \equiv 0$ for $f(x, y, z)=\sum_{p \geq 0} f_{p}(x, y, z)$.

Next we decompose the equation (3) into a series of equations for quasi-homogeneous polynomials.

$$
P u_{p}(x, y, z)=f_{p+1}(x, y, z), \quad p=0,1,2, \ldots
$$


From now on, we research on the conditions for each $f_{p+1}(x, y, z)$ in order that $f_{p+1}(x, y, z) \in \operatorname{Im}\left(P ; \mathcal{O}_{X}\right)$. We write the expansions of $u_{p}$ and $f_{p}$ by

$$
u_{p}(x, y, z)=\sum_{i+2 j=p} u_{i, j}(x) y^{i} z^{j} \text { and } f_{p}(x, y, z)=\sum_{i+2 j=p} f_{i, j}(x) y^{i} z^{j} .
$$

The case $p=0$. Since $u_{0}(x, y, z)=u_{0,0}(x)$ and $f_{1}(x, y, z)=f_{1,0}(x) y$, we have

$$
P u_{0}=u_{0,0}^{\prime}(x) y=f_{1,0}(x) y, \text { that is, } u_{0,0}^{\prime}(x)=f_{1,0}(x) .
$$

This implies $u_{0,0}(x)=C_{0,0}+D_{x}^{-1} f_{1,0}(x)$ where $C_{0,0} \in \mathbb{C}$ denotes the Cauchy data at $x=0$ and $D_{x}^{-1}:=\int_{0}^{x}$ denotes the integration from 0 to $x$.

The case $p=1$. Since $u_{1}(x, y, z)=u_{1,0}(x) y$ and $f_{2}(x, y, z)=f_{2,0}(x) y^{2}+f_{0,1}(x) z$, we have

$$
P u_{1}=u_{1,0}^{\prime}(x) y^{2}-u_{1,0}(x) z=f_{2,0}(x) y^{2}+f_{0,1}(x) z,
$$

that is,

$$
\left\{\begin{array}{l}
u_{1,0}^{\prime}(x)=f_{2,0}(x), \\
-u_{1,0}(x)=f_{0,1}(x)
\end{array} \Leftrightarrow\left(\begin{array}{c}
-D_{x} \\
1
\end{array}\right) u_{1,0}(x)=-\left(\begin{array}{c}
f_{2,0}(x) \\
f_{0,1}(x)
\end{array}\right) .\right.
$$

This implies a compatibility condition

$$
f_{2,0}(x)+f_{0,1}^{\prime}(x) \equiv 0
$$

and $u_{1,0}(x)=-f_{0,1}(x)$ which is uniquely determined.

The case for $p$ is even, that is, $p=2 n(n \geq 1)$. Since

$$
\begin{aligned}
u_{2 n}(x, y, z) & =\sum_{i+2 j=2 n} u_{i, j}(x) y^{i} z^{j} \\
& =u_{2 n, 0}(x) y^{2 n}+u_{2 n-2,1}(x) y^{2 n-2} z+u_{2 n-4,2}(x) y^{2 n-4} z^{2}+\ldots+u_{0, n}(x) z^{n}
\end{aligned}
$$

and

$$
\begin{aligned}
& f_{2 n+1}(x, y, z)=\sum_{i+2 j=2 n+1} f_{i, j}(x) y^{i} z^{j} \\
& \quad=f_{2 n+1,0}(x) y^{2 n+1}+f_{2 n-1,1}(x) y^{2 n-1} z+f_{2 n-3,2}(x) y^{2 n-3} z^{2}+\ldots+f_{1, n}(x) y z^{n},
\end{aligned}
$$

we have

$$
\begin{aligned}
& P u_{2 n}(x, y, z)=y \partial_{x}\left(u_{2 n, 0}(x) y^{2 n}+u_{2 n-2,1}(x) y^{2 n-2} z\right. \\
& \left.+u_{2 n-4,2}(x) y^{2 n-4} z^{2}+\ldots+u_{0, n}(x) z^{n}\right) \\
& -z \partial_{y}\left(u_{2 n, 0}(x) y^{2 n}+u_{2 n-2,1}(x) y^{2 n-2} z\right. \\
& \left.\quad+u_{2 n-4,2}(x) y^{2 n-4} z^{2}+\ldots+u_{0, n}(x) z^{n}\right) \\
& =u_{2 n, 0}^{\prime}(x) y^{2 n+1}+u_{2 n-2,1}^{\prime}(x) y^{2 n-1} z \\
& +u_{2 n-4,2}^{\prime}(x) y^{2 n-3} z^{2}+\ldots+u_{0, n}^{\prime}(x) y z^{n} \\
& -2 n u_{2 n, 0}(x) y^{2 n-1} z-(2 n-2) u_{2 n-2,1}(x) y^{2 n-3} z^{2} \\
& \quad-(2 n-4) u_{2 n-4,2}(x) y^{2 n-5} z^{3}-\ldots-2 u_{2, n-1}(x) y z^{n} \\
& =f_{2 n+1,0}(x) y^{2 n+1}+f_{2 n-1,1}(x) y^{2 n-1} z \\
& +f_{2 n-3,2}(x) y^{2 n-3} z^{2}+\ldots+f_{1, n}(x) y z^{n} .
\end{aligned}
$$


The last expression leads us to the following system of differential equations.

$$
\left\{\begin{aligned}
u_{2 n, 0}^{\prime}(x) & =f_{2 n+1,0}(x), \\
u_{2 n-2,1}^{\prime}(x)-2 n u_{2 n, 0}(x) & =f_{2 n-1,1}(x), \\
& \vdots \\
u_{0, n}^{\prime}(x)-2 u_{2, n-1}(x) & =f_{1, n}(x) .
\end{aligned}\right.
$$

This system is rewritten by the matrix form as follows.

$$
\left(\begin{array}{cccccc}
D_{x} & 0 & \ldots & \cdots & \ldots & 0 \\
-2 n & D_{x} & 0 & \ldots & \ldots & 0 \\
0 & -2 n+2 & D_{x} & 0 & \ldots & 0 \\
\vdots & \ddots & \ddots & \ddots & \ddots & \vdots \\
0 & \cdots & 0 & -4 & D_{x} & 0 \\
0 & \cdots & \cdots & 0 & -2 & D_{x}
\end{array}\right)\left(\begin{array}{c}
u_{2 n, 0} \\
u_{2 n-2,1} \\
u_{2 n-4,2} \\
\vdots \\
u_{2, n-1} \\
u_{0, n}
\end{array}\right)=\left(\begin{array}{c}
f_{2 n+1,0} \\
f_{2 n-1,1} \\
f_{2 n-3,2} \\
\vdots \\
f_{3, n-1} \\
f_{1, n}
\end{array}\right)
$$

The size of matrix differential operator is $(n+1) \times(n+1)$. Therefore, by giving the Cauchy data $\left\{u_{2 n-2 k, k}(0)\right\}_{k=0, \ldots, n}$ at $x=0,\left\{u_{2 n-2 k, k}(x)\right\}$ are uniquely determined by repeated integrations.

The case for $p$ is odd, that is, $p=2 n+1(n \geq 1)$. By the same argument as above, the coefficients $\left\{u_{2 n-2 k+1, k}(x)\right\}_{k=0, \ldots, n}$ satisfy the following matrix relation.

$$
\left(\begin{array}{cccccc}
D_{x} & 0 & \cdots & \ldots & \ldots & 0 \\
-2 n-1 & D_{x} & 0 & \ldots & \ldots & 0 \\
0 & -2 n+1 & D_{x} & 0 & \ldots & 0 \\
\vdots & \ddots & \ddots & \ddots & \ddots & \vdots \\
0 & \ldots & 0 & -5 & D_{x} & 0 \\
0 & \cdots & \cdots & 0 & -3 & D_{x} \\
0 & \ldots & \ldots & 0 & 0 & -1
\end{array}\right)\left(\begin{array}{c}
u_{2 n+1,0} \\
u_{2 n-1,1} \\
u_{2 n-3,2} \\
\vdots \\
u_{3, n-1} \\
u_{1, n}
\end{array}\right)=\left(\begin{array}{c}
f_{2 n+2,0} \\
f_{2 n, 1} \\
f_{2 n-2,2} \\
\vdots \\
f_{4, n-1} \\
f_{2, n} \\
f_{0, n+1}
\end{array}\right) .
$$

The size of matrix differential operator is $(n+2) \times(n+1)$. In order to determine the coefficients $\left\{u_{2 n-2 k+1, k}(x)\right\}$ of formal solution, we must assume the compatibility conditions.

The $(n+2)$-th row equation is $-u_{1, n}(x)=f_{0, n+1}(x)$. Therefore, the coefficient $u_{1, n}(x)$ is determined by $u_{1, n}(x)=-f_{0, n+1}(x)$ uniquely. Next, the coefficient $u_{3, n-1}(x)$ is determined by $u_{3, n-1}(x)=\left(u_{1, n}^{\prime}(x)-f_{2, n}(x)\right) / 3=\left(-f_{0, n+1}^{\prime}(x)-f_{2, n}(x)\right) / 3$. By repeating this argument, we can determine $\left\{u_{2 n-2 k+1, k}(x)\right\}_{k=0, \ldots, n}$ from the equations except the first row. However, $\left\{u_{2 n-2 k+1, k}(x)\right\}_{k=0, \ldots, n}$ must satisfy the first row equation $u_{2 n+1,0}^{\prime}(x)=f_{2 n+2,0}(x)$. By the careful calculation, this is rewritten by

$$
\sum_{k=0}^{n+1} \frac{(2 k-1) ! !}{(2 n+1) ! !} D_{x}^{n-k+1} f_{2 k, n-k+1}(x)=0
$$

which gives the compatibility condition (6). 
2.2. Research on $\operatorname{Ker}\left(\boldsymbol{P} ; \mathcal{O}_{X}\right)$. In this subsection, we calculate explicitly the kernel of the mapping $P: \mathcal{O}_{x}[[y, z]] \rightarrow \mathcal{O}_{x}[[y, z]]$. We consider the equation

$$
P u(X)=0 \Longleftrightarrow P u_{p}(X)=0
$$

for $p=0,1,2, \ldots$.

First we note that if $p$ is odd, then we have $u_{p}(x, y, z) \equiv 0$ from 16 .

In the case when $p=2 n$, the matrix representation is as follows.

$$
\left(\begin{array}{cccccc}
D_{x} & 0 & \cdots & \cdots & \cdots & 0 \\
-2 n & D_{x} & 0 & \cdots & \cdots & 0 \\
0 & -2 n+2 & D_{x} & 0 & \cdots & 0 \\
\vdots & \ddots & \ddots & \ddots & \ddots & \vdots \\
0 & \cdots & 0 & -4 & D_{x} & 0 \\
0 & \cdots & \ldots & 0 & -2 & D_{x}
\end{array}\right)\left(\begin{array}{c}
u_{2 n, 0} \\
u_{2 n-2,1} \\
u_{2 n-4,2} \\
\vdots \\
u_{2, n-1} \\
u_{0, n}
\end{array}\right)=\left(\begin{array}{c}
0 \\
0 \\
0 \\
\vdots \\
0 \\
0
\end{array}\right)
$$

The first row equation is solved as $u_{2 n, 0}(x)=C_{2 n, 0}$. The second row equation is solved as $u_{2 n-2,1}(x)=C_{2 n-2,1}+2 n C_{2 n, 0} x$, and the third equation is solved as $u_{2 n-4,2}(x)=$ $C_{2 n-4,2}+(2 n-2) C_{2 n-2,1} x+2 n(2 n-2) C_{2 n, 0} x^{2} / 2$. We repeat these observations, we have the following relation by the careful calculations.

$$
u_{2 n-2 k, k}(x)=\sum_{\ell=0}^{k} C_{2 n-2 \ell, \ell} \frac{(n-\ell) !}{(n-k) !(k-\ell) !}(2 x)^{k-\ell}
$$

with $u_{2 n-2 \ell, \ell}(0)=C_{2 n-2 \ell, \ell} \in \mathbb{C}$ which is the Cauchy data. Therefore,

$$
\begin{aligned}
u_{2 n}(x, y, z) & =\sum_{k=0}^{n} u_{2 n-2 k, k}(x) y^{2 n-2 k} z^{k} \\
& =\sum_{k=0}^{n} \sum_{\ell=0}^{k} C_{2 n-2 \ell, \ell} \frac{(n-\ell) !}{(n-k) !(k-\ell) !}(2 x)^{k-\ell} y^{2 n-2 k} z^{k} \\
& =\sum_{\ell=0}^{n} C_{2 n-2 \ell, \ell}\left(\sum_{k=\ell}^{n} \frac{(n-\ell) !}{(n-k) !(k-\ell) !}\left(y^{2}\right)^{n-k}(2 x z)^{k-\ell}\right) z^{\ell} \\
& =\sum_{\ell=0}^{n} C_{2 n-2 \ell, \ell}\left(y^{2}+2 x z\right)^{n-\ell} z^{\ell} .
\end{aligned}
$$

By these observations we have the following proposition which includes a part of Main Theorem 1.3(ii).

\section{Proposition 2.1.}

(i) The kernel of the mapping $P: \mathcal{O}_{x}[[y, z]] \rightarrow \mathcal{O}_{x}[[y, z]]$ has an infinite dimensional basis $\left\{\left(y^{2}+2 x z\right)^{n-\ell} z^{\ell}: 0 \leq \ell \leq n, n=0,1,2, \ldots\right\}$.

(ii) The kernel of the mapping $P: \mathcal{O}_{X} \rightarrow \mathcal{O}_{X}$ is isomorphic to the analytic functions

$$
\mathcal{K}:=\left\{v(y, z)=\sum_{n=0}^{\infty} \sum_{k=0}^{n} C_{2 n-2 k, k} y^{2 n-2 k} z^{k} \in \mathcal{O}_{y, z}\right\},
$$


and for $v(y, z) \in \mathcal{K}$, the corresponding kernel is given by

$$
u(x, y, z)=\sum_{n=0}^{\infty} \sum_{k=0}^{n} C_{2 n-2 k, k}\left(y^{2}+2 x z\right)^{n-k} z^{k} \in \mathcal{O}_{X} .
$$

2.3. Convergence of formal solutions. We decompose the formal solution $u$ into $u=u_{\mathrm{e}}+u_{\mathrm{o}}$ by the even part $u_{\mathrm{e}}$ and the odd part $u_{\mathrm{o}}$. Then for $f(x, y, z) \in \mathcal{O}_{X}$, which satisfies the compatibility conditions (6), the following Cauchy problem has a unique formal solution $u(x, y, z) \in \mathcal{O}_{x}[[y, z]]$.

$$
\begin{cases}P u=f(x, y, z) & (f \text { satisfies } 6) \\ u_{\mathrm{e}}(0, y, z)=v(y, z) \in \mathcal{K} & (\text { see }(8)) .\end{cases}
$$

By putting $U(x, y, z)=u(x, y, z)-v(y, z)$ as a new unknown function, we may assume that $v(y, z) \equiv 0$ for the Cauchy data. Namely, in the following, we consider the equation

$$
\left\{\begin{array}{l}
P u=f(x, y, z) \quad(f \text { satisfies }(6)) \\
u_{\mathrm{e}}(0, y, z) \equiv 0
\end{array}\right.
$$

By the matrix representation (15), we have the following expressions for zero Cauchy data.

$$
\begin{aligned}
u_{2 n, 0}(x) & =D_{x}^{-1} f_{2 n+1,0}(x), \\
u_{2 n-2,1}(x) & =2 n D_{x}^{-2} f_{2 n+1,0}(x)+D_{x}^{-1} f_{2 n-1,1}(x), \\
u_{2 n-4,2}(x) & =2^{2} n(n-1) D_{x}^{-3} f_{2 n+1,0}(x)+2(n-1) D_{x}^{-2} f_{2 n-1,1}(x)+D_{x}^{-1} f_{2 n-3,2}(x),
\end{aligned}
$$

and in general

$$
u_{2 n-2 k, k}(x)=\sum_{\ell=0}^{k} 2^{k-\ell} \frac{(n-\ell) !}{(n-k) !} D_{x}^{\ell-k-1} f_{2(n-\ell)+1, \ell}(x),
$$

where $\ell-k-1<0$. Therefore $u_{2 n}(x, y, z)$ is given by

$$
u_{2 n}(x, y, z)=\sum_{k=0}^{n} \sum_{\ell=0}^{k} 2^{k-\ell} \frac{(n-\ell) !}{(n-k) !} D_{x}^{\ell-k-1} f_{2(n-\ell)+1, \ell}(x) y^{2 n-2 k} z^{k} .
$$

Here we notice that $f(x, y, z) \in \mathcal{O}_{X}$ by the assumption, we may assume

$$
\sup _{|x| \leq r}\left|f_{j, k}(x)\right| \leq C A^{j+2 k}, \quad j, k=0,1,2, \ldots,
$$

by some positive constants $C$ and $A$, where $r$ is some fixed positive constant.

By using this estimate, we easily have the following inequality on $|x| \leq r$ :

$$
\left|D_{x}^{-(k-\ell+1)} f_{2(n-\ell)+1, \ell}(x)\right| \leq C A^{2 n+1} \frac{|x|^{k-\ell+1}}{(k-\ell+1) !} .
$$

By this inequality, 25 is estimated as follows.

$$
\begin{aligned}
\left|u_{2 n}(x, y, z)\right| & \leq \sum_{k=0}^{n} \sum_{\ell=0}^{k} 2^{k-\ell} \frac{(n-\ell) !}{(n-k) !}\left|D_{x}^{\ell-k-1} f_{2(n-\ell)+1, \ell}(x)\right||y|^{2 n-2 k}|z|^{k} \\
& \leq \sum_{k=0}^{n} \sum_{\ell=0}^{k} 2^{k-\ell} \frac{(n-\ell) !}{(n-k) !} C A^{2 n+1} \frac{|x|^{k-\ell+1}}{(k-\ell+1) !}|y|^{2 n-2 k}|z|^{k}
\end{aligned}
$$




$$
\begin{aligned}
& \leq C A^{2 n+1}|x| \sum_{k=0}^{n} \sum_{\ell=0}^{k} \frac{(n-\ell) !}{(n-k) !(k-\ell) !}\left(|y|^{2}\right)^{n-k}(2|x z|)^{k-\ell}|z|^{\ell} \\
& =C A^{2 n+1}|x| \sum_{\ell=0}^{n} \sum_{i=0}^{n-\ell} \frac{(n-\ell) !}{(n-\ell-i) ! i !}\left(|y|^{2}\right)^{n-\ell-i}(2|x z|)^{i}|z|^{\ell} \\
& =C A^{2 n+1}|x| \sum_{\ell=0}^{n}\left(|y|^{2}+2|x z|\right)^{n-\ell}|z|^{\ell} \\
& \leq C A^{2 n+1}|x|\left(|y|^{2}+2|x z|+|z|\right)^{n} .
\end{aligned}
$$

This shows the convergence of the even part $u_{\mathrm{e}}(x, y, z)=\sum_{n=0}^{\infty} u_{2 n}(x, y, z)$ in a neighborhood of the origin.

Next, we estimate the odd part $u_{\mathrm{o}}(x, y, z)=\sum_{n=0}^{\infty} u_{2 n+1}(x, y, z)$. By the matrix representation (16), we have the following expressions.

$$
\begin{aligned}
u_{1, n}(x) & =-f_{0, n+1}(x), \\
u_{3, n-1}(x) & =-\frac{1}{3} f_{2, n}(x)-\frac{1}{3} D_{x} f_{0, n+1}(x), \\
u_{5, n-1}(x) & =-\frac{1}{5} f_{4, n-1}(x)-\frac{1}{5 \cdot 3} D_{x} f_{2, n}(x)-\frac{1}{5 \cdot 3} D_{x}^{2} f_{0, n+1}(x),
\end{aligned}
$$

and in general

$$
u_{2 k+1, n-k}(x)=-\sum_{\ell=0}^{k} \frac{(2(k-\ell)-1) ! !}{(2 k+1) ! !} D_{x}^{\ell} f_{2(k-\ell), n-k+\ell+1}(x) .
$$

Therefore, $u_{2 n+1}(x, y, z)$ is given by

$$
\begin{aligned}
u_{2 n+1}(x, y, z) & =\sum_{k=0}^{n} u_{2 k+1, n-k}(x) y^{2 k+1} z^{n-k} \\
& =-\sum_{k=0}^{n} \sum_{\ell=0}^{k} \frac{(2(k-\ell)-1) ! !}{(2 k+1) ! !} D_{x}^{\ell} f_{2(k-\ell), n-k+\ell+1}(x) y^{2 k+1} z^{n-k} .
\end{aligned}
$$

Here we take and fix $r^{\prime}$ satisfying $0<r^{\prime}<r$ and $r-r^{\prime}<1$. Since

$$
\sup _{|x| \leq r}\left|f_{2(k-\ell), n-k+\ell+1}(x)\right| \leq C A^{2 n+2}
$$

(see $(26)$ ), we have the following estimate by using the Cauchy integral formula.

For $|x| \leq r^{\prime}$,

$$
\begin{aligned}
\left|D_{x}^{\ell} f_{2(k-\ell), n-k+\ell+1}(x)\right| & =\left|\frac{\ell !}{2 \pi i} \oint_{|z-x|=r-r^{\prime}} \frac{f_{2(k-\ell), n-k+\ell+1}(z)}{(z-x)^{\ell+1}} d z\right| \\
& \leq \frac{\ell !}{2 \pi} \oint_{|z-x|=r-r^{\prime}} \frac{C A^{2 n+2}}{\left(r-r^{\prime}\right)^{\ell+1}}|d z| \\
& =\ell ! \times C A^{2 n+2}\left(\frac{1}{r-r^{\prime}}\right)^{\ell}=: C A^{2 n+2} B^{\ell} \ell !
\end{aligned}
$$

where $B=1 /\left(r-r^{\prime}\right)$ is a constant greater than 1 . 
By using this inequality, we have the following estimate on $|x| \leq r^{\prime}$ for $u_{2 k+1, n-k}(x)$ :

$$
\begin{aligned}
\left|u_{2 k+1, n-k}(x)\right| & \leq \sum_{\ell=0}^{k} \frac{(2(k-\ell)-1) ! !}{(2 k+1) ! !}\left|D_{x}^{\ell} f_{2(k-\ell), n-k+\ell+1}(x)\right| \\
& \leq C A^{2 n+2} \sum_{\ell=0}^{k} \frac{(2(k-\ell)-1) ! ! \ell !}{(2 k+1) ! !} B^{\ell} .
\end{aligned}
$$

By an easy calculation, we get the estimate $\frac{(2(k-\ell)-1) ! ! \ell !}{(2 k+1) ! !} \leq 1$. Therefore,

$$
\begin{aligned}
\left|u_{2 k+1, n-k}(x)\right| & \leq C A^{2 n+2} \sum_{\ell=0}^{k} \frac{(2(k-\ell)-1) ! ! \ell !}{(2 k+1) ! !} B^{\ell} \\
& \leq C A^{2 n+2} \sum_{\ell=0}^{k} B^{\ell}=C A^{2 n+2} \frac{B^{k+1}-1}{B-1}<\frac{C A^{2 n+2}}{B-1} \times B^{k+1} .
\end{aligned}
$$

Hence we can estimate for $u_{2 n+1}(x, y, z)=\sum_{k=0}^{n} u_{2 k+1, n-k}(x) y^{2 k+1} z^{n-k}$ on $|x| \leq r^{\prime}$ as follows.

$$
\begin{aligned}
\left|u_{2 n+1}(x, y, z)\right| & \leq \sum_{k=0}^{n}\left|u_{2 k+1, n-k}(x)\right||y|^{2 k+1}|z|^{n-k} \leq \sum_{k=0}^{n} \frac{C A^{2 n+2}}{B-1} B^{k+1}|y|^{2 k+1}|z|^{n-k} \\
& =\frac{C A^{2 n+2} B|y|}{B-1} \sum_{k=0}^{n}\left(B|y|^{2}\right)^{k}|z|^{n-k}<\frac{C A^{2 n+2} B|y|}{B-1}\left(B|y|^{2}+|z|\right)^{n} .
\end{aligned}
$$

Therefore, $u_{\mathrm{o}}(x, y, z)$ is convergent on $|x| \leq r^{\prime}$ and in a neighborhood of $(y, z)=(0,0)$.

This completes the proof of Main Theorem 1.3 .

\section{References}

[GT] R. Gérard, H. Tahara, Singular Nonlinear Partial Differential Equations, Aspects Math., Friedr. Vieweg and Sohn, Braunschweig, 1996.

[H] M. Hibino, Divergence property of formal solutions for singular first order linear partial differential equations, Publ. Res. Inst. Math. Sci. 35 (1999), 893-919.

[MS1] M. Miyake, A. Shirai, Convergence of formal solutions of first order singular nonlinear partial differential equations in the complex domain, Ann. Polon. Math. 74 (2000), $215-228$.

[MS2] M. Miyake, A. Shirai, Structure of formal solutions of nonlinear first order singular partial differential equations in complex domain, Funkcial. Ekvac. 48 (2005), 113-136.

[O] T. Oshima, On the theorem of Cauchy-Kowalevsky for first order linear differential equations with degenerate principal symbols, Proc. Japan Acad. 49 (1973), 83-87.

[S] A. Shirai, A Maillet type theorem for first order singular nonlinear partial differential equations, Publ. Res. Inst. Math. Sci. 39 (2003), 275-296. 
